\title{
The state complexity of star-complement-star
}

\author{
Galina Jiráskováa ${ }^{1, \star}$ and Jeffrey Shallit ${ }^{2}$ \\ 1 Mathematical Institute, Slovak Academy of Sciences \\ Grešákova 6, 04001 Košice, Slovakia \\ jiraskov@saske.sk \\ 2 School of Computer Science, University of Waterloo \\ Waterloo, ON N2L 3G1 Canada \\ shallit@cs.uwaterloo.ca
}

\begin{abstract}
We resolve an open question by determining matching (asymptotic) upper and lower bounds on the state complexity of the operation that sends a language $L$ to $\left(\overline{L^{*}}\right)^{*}$.
\end{abstract}

\section{Introduction}

Let $\Sigma$ be a finite nonempty alphabet, let $L \subseteq \Sigma^{*}$ be a language, let $\bar{L}=\Sigma^{*}-L$ denote the complement of $L$, and let $L^{*}$ (resp., $L^{+}$) denote the Kleene closure (resp., positive closure) of the language $L$. If $L$ is a regular language, its state complexity is defined to be the number of states in the minimal deterministic finite automaton accepting $L$ [7. In this paper we resolve an open question by determining matching (asymptotic) upper and lower bounds on the deterministic state complexity of the operations

$$
\begin{aligned}
& L \rightarrow\left(\overline{L^{*}}\right)^{*} \\
& L \rightarrow\left(\overline{L^{+}}\right)^{+} .
\end{aligned}
$$

To simplify the exposition, we will write everything using an exponent notation, using $c$ to represent complement, as follows:

$$
\begin{aligned}
L^{+c} & :=\overline{L^{+}} \\
L^{+c+} & :=\left(\overline{L^{+}}\right)^{+},
\end{aligned}
$$

and similarly for $L^{* c}$ and $L^{* c *}$.

Note that

$$
L^{* c *}= \begin{cases}L^{+c+}, & \text { if } \varepsilon \notin L ; \\ L^{+c+} \cup\{\varepsilon\}, & \text { if } \varepsilon \in L .\end{cases}
$$

It follows that the state complexity of $L^{+c+}$ and $L^{* c *}$ differ by at most 1 . In what follows, we will work only with $L^{+c+}$.

\footnotetext{
* Research supported by VEGA grant 2/0183/11.
} 


\section{Upper Bound}

Consider a deterministic finite automaton (DFA) $D=\left(Q_{n}, \Sigma, \delta, 0, F\right)$ accepting a language $L$, where $Q_{n}:=\{0,1, \ldots, n-1\}$. As an example, consider the threestate DFA over $\{a, b, c, d\}$ shown in Fig. 1 (left). To get a nondeterministic finite automaton (NFA) $N_{1}$ for the language $L^{+}$from the DFA $D$, we add an $\varepsilon$ transition from every non-initial final state to the state 0 . In our example, we add an $\varepsilon$-transition from state 1 to state 0; see Fig. 1 (right). After applying the subset construction to the NFA $N_{1}$ we get a DFA $D_{1}$ for the language $L^{+}$. The state set of $D_{1}$ consists of subsets of $Q_{n}$ see Fig. 2 (left). Here the sets in the labels of states are written without commas and brackets; thus, for example 012 stands for the set $\{0,1,2\}$. Next, we interchange the roles of the final and non-final states of the DFA $D_{1}$, and get a DFA $D_{2}$ for the language $L^{+c}$; see Fig. 2 (right).

To get an NFA $N_{3}$ for $L^{+c+}$ from the DFA $D_{2}$, we add an $\varepsilon$-transition from each non-initial final state of $D_{2}$ to the state $\{0\}$, see Fig. 3 (top). Applying the subset construction to the NFA $N_{3}$ results in a DFA $D_{3}$ for the language $L^{+c+}$ with its state set consisting of some sets of subsets of $Q_{n}$; see Fig. 3 (middle). Here, for example, the label 0,2 corresponds to the set $\{\{0\},\{2\}\}$. This gives an upper bound of $2^{2^{n}}$ on the state complexity of the operation plus-complementplus.

Our first result shows that in the minimal DFA for $L^{+c+}$ we do not have any state $\left\{S_{1}, S_{2}, \ldots, S_{k}\right\}$, in which a set $S_{i}$ is a subset of some other set $S_{j}$; see Fig. 3 (bottom). This reduces the upper bound to the number of antichains of subsets of an $n$-element set known as the Dedekind number $M(n)$ with [2]

$$
\left(\begin{array}{c}
n \\
\lfloor n / 2\rfloor
\end{array}\right) \leq \log M(n) \leq\left(\begin{array}{c}
n \\
\lfloor n / 2\rfloor
\end{array}\right)\left(1+O\left(\frac{\log n}{n}\right)\right) .
$$
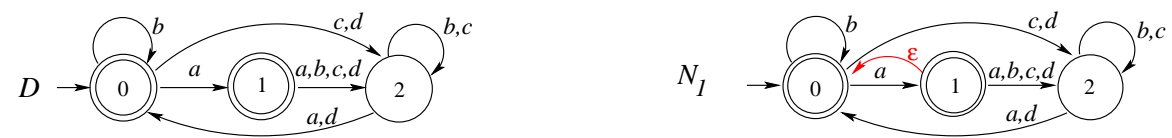

Fig. 1. DFA $D$ for a language $L$ and NFA $N_{1}$ for the language $L^{+}$.
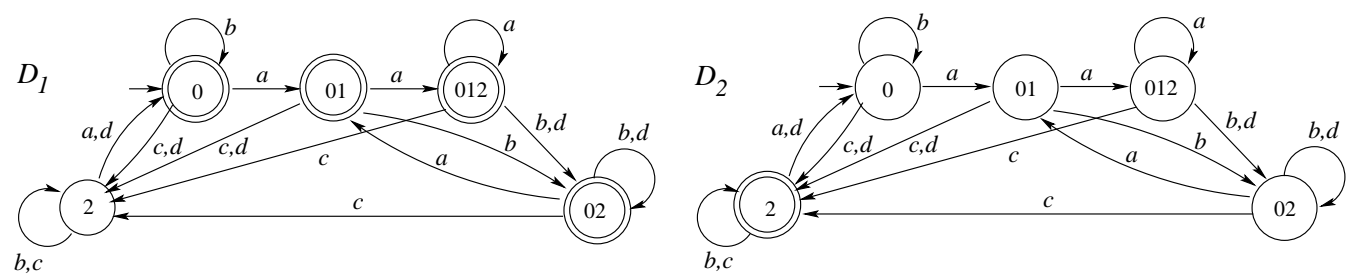

Fig. 2. DFA $D_{1}$ for language $L^{+}$and DFA $D_{2}$ for the language $L^{+c}$. 

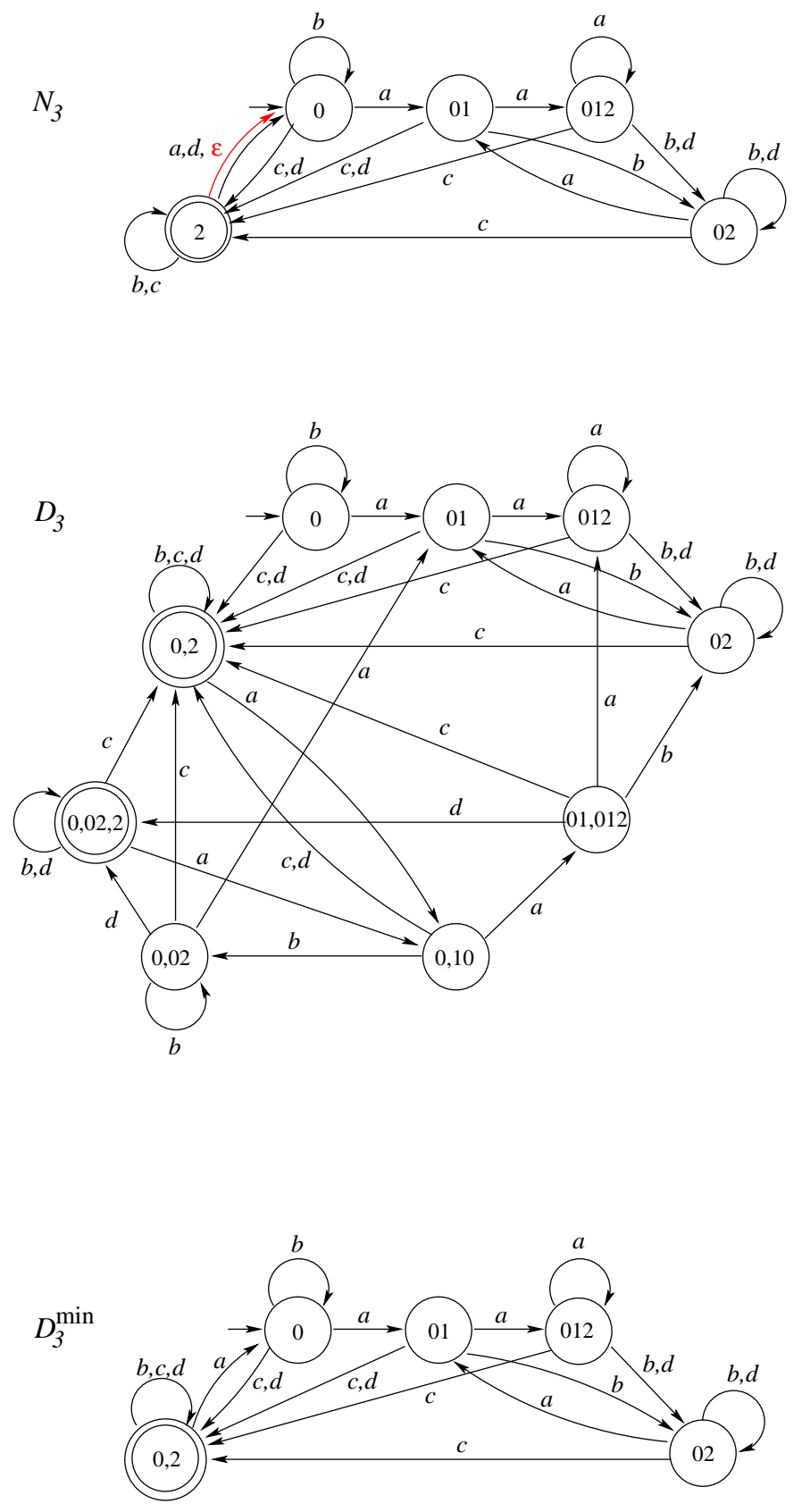

Fig. 3. NFA $N_{3}$, DFA $D_{3}$, and the minimal DFA $D_{3}^{\min }$ for the language $L^{+c+}$. 
Lemma 1. If $S$ and $T$ are subsets of $Q_{n}$ such that $S \subseteq T$, then the states $\{S, T\}$ and $\{S\}$ of the DFA $D_{3}$ for the language $L^{+c+}$ are equivalent.

Proof. Let $S$ and $T$ be subsets of $Q_{n}$ such that $S \subseteq T$. We only need to show that if a string $w$ is accepted by the NFA $N_{3}$ starting from the state $T$, then it also is accepted by $N_{3}$ from the state $S$.

Assume $w$ is accepted by $N_{3}$ from $T$. Then in the NFA $N_{3}$, an accepting computation on $w$ from state $T$ looks like this:

$$
T \stackrel{u}{\rightarrow} T_{1} \stackrel{\varepsilon}{\rightarrow}\{0\} \stackrel{v}{\rightarrow} T_{2},
$$

where $w=u v$, and state $T$ goes to an accepting state $T_{1}$ on $u$ without using any $\varepsilon$-transitions, then $T_{1}$ goes to $\{0\}$ on $\varepsilon$, and then $\{0\}$ goes to an accepting state $T_{2}$ on $v$; it also may happen that $w=u$, in which case the computation ends in $T_{1}$. Let us show that $S$ goes to an accepting state of the NFA $N_{3}$ on $u$.

Since $T$ goes to an accepting state $T_{1}$ on $u$ in the NFA $N_{3}$ without using any $\varepsilon$-transition, state $T$ goes to the accepting state $T_{1}$ in the DFA $D_{2}$, and therefore to the rejecting state $T_{1}$ of the DFA $D_{1}$. Thus, every state $q$ in $T$ goes to rejecting states in the NFA $N_{1}$. Since $S \subseteq T$, every state in $S$ goes to rejecting states in the NFA $N_{1}$, and therefore $S$ goes to a rejecting state $S_{1}$ in the DFA $D_{1}$, thus to the accepting state $S_{1}$ in the DFA $D_{2}$. Hence $w=u v$ is accepted from $S$ in the NFA $N_{3}$ by computation

$$
S \stackrel{u}{\rightarrow} S_{1} \stackrel{\varepsilon}{\rightarrow}\{0\} \stackrel{v}{\rightarrow} T_{2} .
$$

Hence whenever a state $\mathcal{S}=\left\{S_{1}, S_{2}, \ldots, S_{k}\right\}$ of the DFA $D_{3}$ contains two subsets $S_{i}$ and $S_{j}$ with $i \neq j$ and $S_{i} \subseteq S_{j}$, then it is equivalet to state $\mathcal{S} \backslash\left\{S_{j}\right\}$. Using this property, we get the following result.

Lemma 2. Let $D$ be a DFA for a language $L$ with state set $Q_{n}$, and $D_{3}^{\min }$ be the minimal DFA for $L^{+c+}$ as described above. Then every state of $D_{3}^{\min }$ can be expressed in the form

$$
\mathcal{S}=\left\{X_{1}, X_{2}, \ldots, X_{k}\right\}
$$

where

$-1 \leq k \leq n$;

- there exist subsets $S_{1} \subseteq S_{2} \subseteq \cdots \subseteq S_{k} \subseteq Q_{n}$; and

- there exist $q_{1}, \ldots, q_{k}$, pairwise distinct states of $D$ not in $S_{k}$; such that

- $X_{i}=\left\{q_{i}\right\} \cup S_{i}$ for $i=1,2, \ldots, k$.

Proof. Let $D=\left(Q_{n}, \Sigma, \delta, 0, F\right)$.

For a state $q$ in $Q_{n}$ and a symbol $a$ in $\Sigma$, let $q . a$ denote the state in $Q_{n}$, to which $q$ goes on $a$, that is, $q . a=\delta(q, a)$. For a subset $X$ of $Q_{n}$ let $X . a$ denote the set of states to which states in $X$ go by $a$, that is,

$$
X . a=\bigcup_{q \in X}\{\delta(q, a)\} .
$$


Consider transitions on a symbol $a$ in automata $D, N_{1}, D_{1}, D_{2}, N_{3}$; Fig. 4 illustrates these transitions. In the NFA $N_{1}$, each state $q$ goes to a state in $\{0, q . a\}$ if $q . a$ is a final state of $D$, and to state q.a if $q . a$ is non-final. It follows that in the DFA $D_{1}$ for $L^{+}$, each state $X$ (a subset of $Q_{n}$ ) goes on $a$ to final state $\{0\} \cup X$.a if $X . a$ contains a final state of $D$, and to non-final state $X . a$ if all states in $X . a$ are non-final in $D$. Hence in the DFA $D_{2}$ for $L^{+c}$, each state $X$ goes on $a$ to non-final state $\{0\} \cup X$. $a$ if $X$.a contains a final state of $D$, and to the final state $X . a$ if all states in $X . a$ are non-final in $D$.

Therefore, in the NFA $N_{3}$ for $L^{+c+}$, each state $X$ goes on $a$ to a state in $\{\{0\}, X . a\}$ if all states in $X . a$ are non-final in $D$, and to state $\{0\} \cup X . a$ if $X . a$ contains a final state of $D$.

To prove the lemma for each state, we use induction on the length of the shortest path from the initial state to the state of $D_{3}^{\min }$ in question. The base case is a path of length 0 . In this case, the initial state is $\{\{0\}\}$, which is in the required form (11) with $k=1, q_{1}=0$, and $S_{1}=\emptyset$.
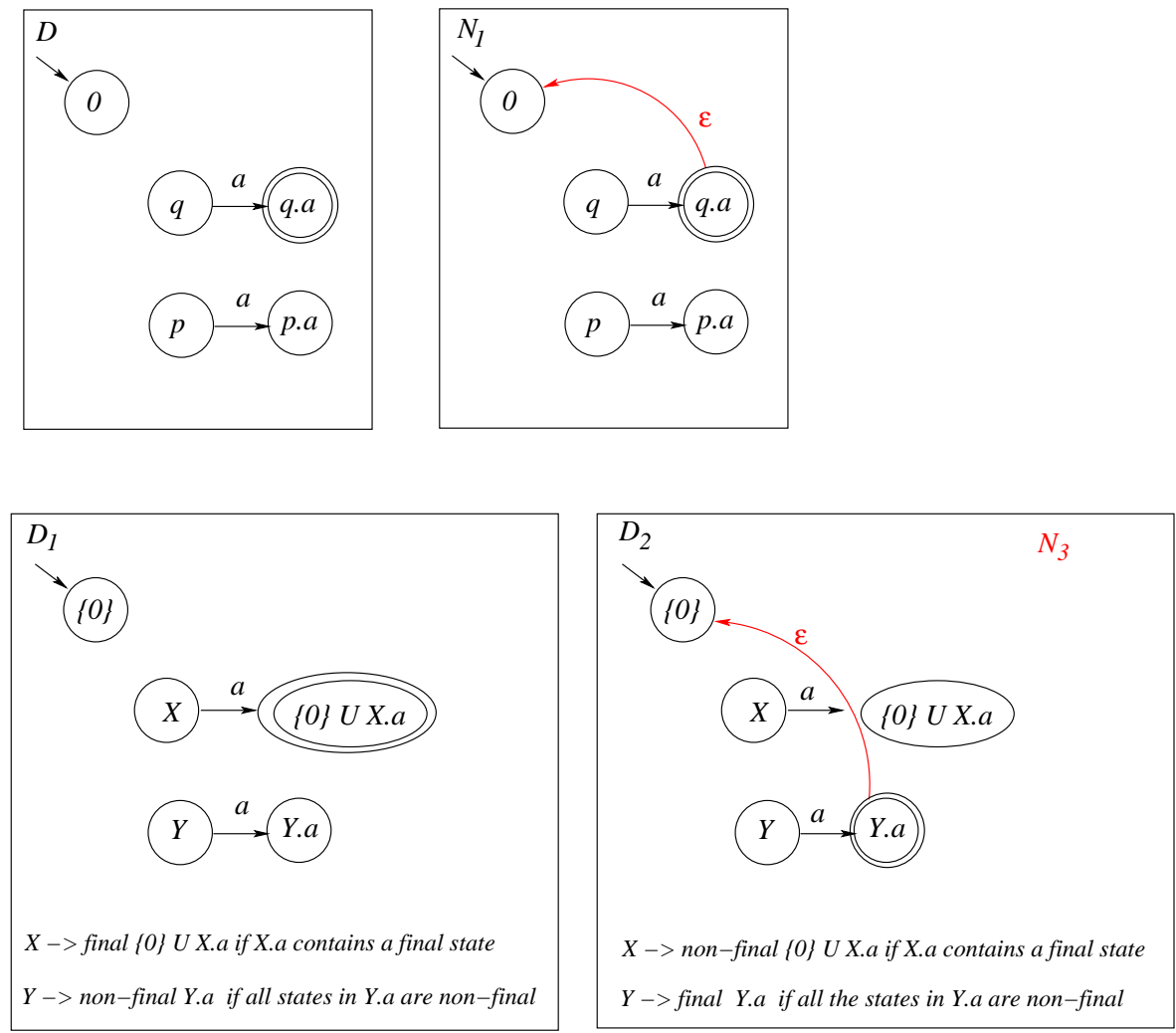

Fig. 4. Transitions under symbol $a$ in automata $D, N_{1}, D_{1}, D_{2}, N_{3}$. 
For the induction step, let

$$
\mathcal{S}=\left\{X_{1}, X_{2}, \ldots, X_{k}\right\}
$$

where $1 \leq k \leq n$, and

- $S_{1} \subseteq S_{2} \subseteq \cdots \subseteq S_{k} \subseteq Q_{n}$,

- $q_{1}, \ldots, q_{k}$ are pairwise distinct states of $D$ that are not in $S_{k}$ and

- $X_{i}=\left\{q_{i}\right\} \cup S_{i}$ for $i=1,2, \ldots, k$.

We now prove the result for all states reachable from $\mathcal{S}$ on a symbol $a$.

First, consider the case that each $X_{i}$ goes on $a$ to a non-final state $X_{i}^{\prime}$ in the NFA $N_{3}$. It follows that $\mathcal{S}$ goes on $a$ to $\mathcal{S}^{\prime}=\left\{X_{1}^{\prime}, X_{2}^{\prime}, \ldots, X_{k}^{\prime}\right\}$, where

$$
X_{i}^{\prime}=\left\{q_{i} . a\right\} \cup S_{i} \cdot a \cup\{0\} .
$$

Write $p_{i}=q_{i} . a$ and $P_{i}=S_{i} . a \cup\{0\}$. Then we have $P_{1} \subseteq P_{2} \subseteq \cdots \subseteq P_{k} \subseteq Q_{n}$.

If $p_{i}=p_{j}$ for some $i, j$ with $i<j$, then $X_{i}^{\prime} \subseteq X_{j}^{\prime}$, and therefore $X_{j}^{\prime}$ can be removed from state $\mathcal{S}^{\prime}$ in the minimal DFA $D_{3}^{\min }$. After several such removals, we arrive at an equivalent state

$$
\mathcal{S}^{\prime \prime}=\left\{X_{1}^{\prime \prime}, X_{2}^{\prime \prime}, \ldots, X_{\ell}^{\prime \prime}\right\}
$$

where $\ell \leq k, X_{i}^{\prime \prime}=\left\{r_{i}\right\} \cup R_{i}$ and the states $r_{1}, r_{2}, \ldots, r_{\ell}$ are pairwise distinct.

If $r_{i} \in R_{\ell}$ for some $i$ with $i<\ell$, then $X_{i} \subseteq R_{\ell}$; thus $R_{\ell}$ can be removed. After all such removals, we get an equivalent set

$$
\mathcal{S}^{\prime \prime \prime}=\left\{X_{1}^{\prime \prime \prime}, X_{2}^{\prime \prime \prime}, \ldots, X_{m}^{\prime \prime \prime}\right\}
$$

where $m \leq \ell, X_{i}^{\prime \prime \prime}=\left\{t_{i}\right\} \cup T_{i}$ and the states $t_{1}, t_{2}, \ldots, t_{m}$ are pairwise distinct and $t_{1}, t_{2}, \ldots, t_{m-1}$ are not in $T_{m}$. If $t_{m} \notin T_{m}$, then the state $\mathcal{S}^{\prime \prime \prime}$ is in the required form (11). Otherwise, if $T_{m-1}$ is a proper subset of $T_{m}$, then there is a state $t$ in $T_{m}-T_{m-1}$, and then we can take $X_{m}^{\prime \prime \prime}=\{t\} \cup T_{m}-\{t\}$ : since $t_{1}, \ldots, t_{m-1}$ are not in $T_{m}$, they are distinct from $t$, and moreover $T_{m-1} \subseteq T_{m}-\{t\}$.

If $T_{m-1}=T_{m}$, then $X_{m-1}^{\prime \prime \prime} \supseteq X_{m}^{\prime \prime \prime}$, and therefore $X_{m-1}^{\prime \prime \prime}$ can be removed from $\mathcal{S}^{\prime \prime \prime}$. After all these removals we either reach some $T_{i}$ that is a proper subset of $T_{m}$, and then pick a state $t$ in $T_{m}-T_{i}$ in the same way as above, or we only get a single set $T_{m}$, which is in the required form $\left\{r_{m}\right\} \cup T_{m}-\left\{r_{m}\right\}$.

This proves that if each $X_{i}$ in $\mathcal{S}$ goes on $a$ to a non-final state $X_{i}^{\prime}$ in the NFA $N_{3}$, then $\mathcal{S}$ goes on $a$ in the DFA $D_{3}^{\min }$ to a set that is in the required form (11).

Now consider the case that at least one $X_{j}$ in $\mathcal{S}$ goes to a final state $X_{j}^{\prime}$ in the NFA $N_{3}$. It follows that $\mathcal{S}$ goes to a final state

$$
\mathcal{S}^{\prime}=\left\{\{0\}, X_{1}^{\prime}, X_{2}^{\prime}, \ldots, X_{k}^{\prime}\right\},
$$

where $X_{j}^{\prime}=\left\{q_{j} . a\right\} \cup S_{j} \cdot a$ and if $i \neq j$, then $X_{i}^{\prime}=\left\{q_{i} \cdot a\right\} \cup S_{i} \cdot a$ or $X_{i}^{\prime}=$ $\{0\} \cup\left\{q_{i} . a\right\} \cup S_{i} . a$ We now can remove all $X_{i}$ that contain state 0 , and arrive at an equivalent state

$$
\mathcal{S}^{\prime \prime}=\left\{\{0\}, X_{1}^{\prime \prime}, X_{2}^{\prime \prime}, \ldots, X_{\ell}^{\prime \prime}\right\}
$$


where $\ell \leq k$, and $X_{i}^{\prime \prime}=\left\{p_{i}\right\} \cup P_{i}$, and $P_{1} \subseteq P_{2} \subseteq \cdots \subseteq P_{\ell} \subseteq Q_{n}$, and each $p_{i}$ is distinct from 0 .

Now in the same way as above we arrive at an equivalent state

$$
\left\{\{0\},\left\{t_{1}\right\} \cup T_{1}, \ldots,\left\{t_{m}\right\} \cup T_{m}\right\}
$$

where $m \leq \ell$, all the $t_{i}$ are pairwise distinct and different from 0 , and moreover, the states $t_{1}, \ldots, t_{m-1}$ are not in $T_{m}$. If $t_{m}$ is not in $T_{m}$, then we are done. Otherwise, we remove all sets with $T_{i}=T_{m}$. We either arrive at a proper subset $T_{j}$ of $T_{m}$, and may pick a state $t$ in $T_{m}-T_{j}$ to play the role of new $t_{m}$, or we arrive at $\left\{\{0\}, T_{m}\right\}$, which is in the required form $\left\{\{0\} \cup \emptyset, t_{m} \cup T_{m}-\left\{t_{m}\right\}\right\}$. This completes the proof of the lemma.

Corollary 1 (Star-Complement-Star: Upper Bound). If a language $L$ is accepted by a DFA of $n$ states, then the language $L^{* * *}$ is accepted by a DFA of $2^{O(n \log n)}$ states.

Proof. Lemma 2 gives the following upper bound

$$
\sum_{k=1}^{n}\left(\begin{array}{l}
n \\
k
\end{array}\right) k !(k+1)^{n-k}
$$

since we first choose any permutation of $k$ distinct elements $q_{1}, \ldots, q_{k}$, and then represent each set $S_{i}$ as disjoint union of sets $S_{1}^{\prime}, S_{2}^{\prime}, \ldots, S_{i}^{\prime}$ given by a function $f$ from $Q_{n}-\left\{q_{1}, \ldots, q_{k}\right\}$ to $\{1,2, \ldots, k+1\}$ as follows:

$$
S_{i}^{\prime}=\{q \mid f(q)=i\}, \quad S_{i}=S_{1}^{\prime} \dot{\cup} S_{2}^{\prime} \dot{\cup} \cdots \dot{\cup} S_{i}^{\prime},
$$

while states with $f(q)=k+1$ will be outside each $S_{i}^{\prime}$; here $\dot{U}$ denotes a disjoint union. Next, we have

$$
\sum_{k=1}^{n}\left(\begin{array}{l}
n \\
k
\end{array}\right) k !(k+1)^{n-k} \leq n ! \sum_{k=1}^{n}\left(\begin{array}{l}
n \\
k
\end{array}\right)(n+1)^{n-k} \leq n !(n+2)^{n}=2^{O(n \log n)}
$$

and the upper bound follows.

Remark 1. The summation $\sum_{k=1}^{n}\left(\begin{array}{l}n \\ k\end{array}\right) k !(k+1)^{n-k}$ differs by one from Sloane's sequence A072597 [5. These numbers are the coefficients of the exponential generating function of $1 /\left(e^{-x}-x\right)$. It follows, by standard techniques, that these numbers are asymptotically given by $C_{1} W(1)^{-n} n$ !, where

$$
W(1) \doteq .5671432904097838729999686622103555497538
$$

is the Lambert $\mathrm{W}$-function evaluated at 1 , equal to the positive real solution of the equation $e^{x}=1 / x$, and $C_{1}$ is a constant, approximately

$$
1.12511909098678593170279439143182676599 .
$$

The convergence is quite fast; this gives a somewhat more explicit version of the upper bound. 


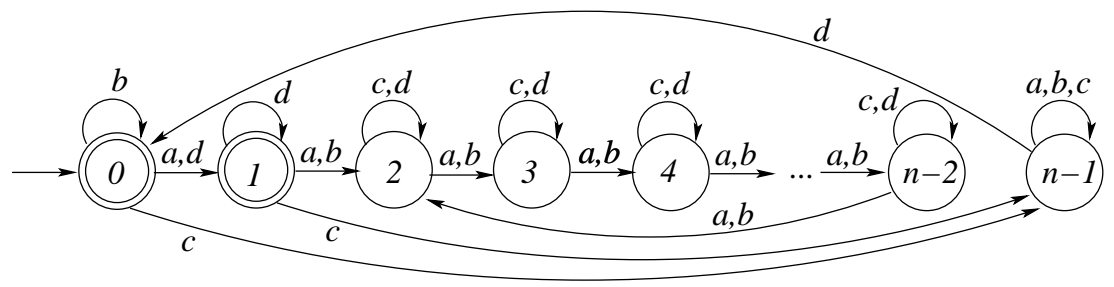

Fig. 5. DFA $D$ over $\{a, b, c, d\}$ with many reachable states in DFA $D_{3}$ for $L^{+c+}$.

\section{Lower Bound}

We now turn to the matching lower bound on the state complexity of pluscomplement-plus. The basic idea is to create one DFA where the DFA for $L^{+c+}$ has many reachable states, and another where the DFA for $L^{+c+}$ has many distinguishable states. Then we "join" them together in Corollary 2

The following lemma uses a four-letter alphabet to prove the reachability of some specific states of the DFA $D_{3}$ for plus-complement-plus.

Lemma 3. There exists an n-state DFA $D=\left(Q_{n},\{a, b, c, d\}, \delta, 0,\{0,1\}\right)$ such that in the DFA $D_{3}$ for the language $L(D)^{+c+}$ every state of the form

$$
\left\{\left\{0, q_{1}\right\} \cup S_{1},\left\{0, q_{2}\right\} \cup S_{2}, \ldots,\left\{0, q_{k}\right\} \cup S_{k}\right\}
$$

is reachable, where $1 \leq k \leq n-2, S_{1}, S_{2}, \ldots, S_{k}$ are subsets of $\{2,3, \ldots, n-2\}$ with $S_{1} \subseteq S_{2} \subseteq \cdots \subseteq S_{k}$, and the $q_{1}, \ldots, q_{k}$ are pairwise distinct states in $\{2,3, \ldots, n-2\}$ that are not in $S_{k}$.

Proof. Consider the DFA $D$ over $\{a, b, c, d\}$ shown in Fig. 5 Let $L$ be the language accepted by the DFA $D$.

Construct the NFA $N_{1}$ for the language $L^{+}$from the DFA $D$ by adding loops on $a$ and $d$ in the initial state 0 . In the subset automaton corresponding to the NFA $N_{1}$, every subset of $\{0,1, \ldots, n-2\}$ containing state 0 is reachable from the initial state $\{0\}$ on a string over $\{a, b\}$ since each subset $\left\{0, i_{1}, i_{2}, \ldots, i_{k}\right\}$ of size $k$, where $1 \leq k \leq n-1$ and $1 \leq i_{1}<i_{2}<\cdots<i_{k} \leq n-2$, is reached from the set $\left\{0, i_{2}-i_{1}, \ldots, i_{k}-i_{1}\right\}$ of size $k-1$ on the string $a b^{i_{1}-1}$. Moreover, after reading every symbol of string $a b^{i_{1}-1}$, the subset automaton is always in a set that contains state 0 . All such states are rejecting in the DFA $D_{2}$ for the language $L^{+c}$, and therefore, in the NFA $N_{3}$ for $L^{+c+}$, the initial state $\{0\}$ only goes to the rejecting state $\left\{0, i_{1}, i_{2}, \ldots, i_{k}\right\}$ on $a b^{i_{1}-1}$.

Hence in the DFA $D_{3}$, for every subset $S$ of $\{0,1, \ldots, n-2\}$ containing 0 , the initial state $\{\{0\}\}$ goes to the state $\{S\}$ on a string $w$ over $\{a, b\}$.

Now notice that transitions on symbols $a$ and $b$ perform the cyclic permutation of states in $\{2,3, \ldots, n-2\}$. For every state $q$ in $\{2,3, \ldots, n-2\}$ and an integer $i$, let

$$
q \ominus i=((q-i-2) \bmod n-3)+2
$$


denote the state in $\{2,3, \ldots, n-2\}$ that goes to the state $q$ on string $a^{i}$, and, in fact, on every string over $\{a, b\}$ of length $i$. Next, for a subset $S$ of $\{2,3, \ldots, n-2\}$ let

$$
S \ominus i=\{q \ominus i \mid q \in S\} .
$$

Thus $S \ominus i$ is a shift of $S$, and if $q \notin S$, then $q \ominus i \notin S \ominus i$.

The proof of the lemma now proceeds by induction on $k$. To prove the base case, let $S_{1}$ be a subset of $\{2,3, \ldots, n-2\}$ and $q_{1}$ be a state in $\{2,3, \ldots, n-2\}$ with $q_{1} \notin S_{1}$. In the NFA $N_{3}$, the initial state $\{0\}$ goes to the state $\{0\} \cup S_{1}$ on a string $w$ over $\{a, b\}$. Next, state $q_{1} \ominus|w|$ is in $\{2,3, \ldots, n-2\}$, and it is reached from state 1 on a string $b^{\ell}$, while state 0 goes to itself on $b$. In the DFA $D_{3}$ we thus have

$$
\{\{0\}\} \stackrel{a}{\rightarrow}\{\{0,1\}\} \stackrel{b^{\ell}}{\rightarrow}\left\{\left\{0, q_{1} \ominus|w|\right\}\right\} \stackrel{w}{\rightarrow}\left\{\left\{0, q_{1}\right\} \cup S_{1}\right\}
$$

which proves the base case.

Now assume that every set of size $k-1$ satisfying the lemma is reachable in the DFA $D_{3}$. Let

$$
\mathcal{S}=\left\{\left\{0, q_{1}\right\} \cup S_{1},\left\{0, q_{2}\right\} \cup S_{2}, \ldots,\left\{0, q_{k}\right\} \cup S_{k}\right\}
$$

be a set of size $k$ satisfying the lemma. Let $w$ be a string, on which $\{\{0\}\}$ goes to $\left\{\{0\} \cup S_{1}\right\}$, and let $\ell$ be an integer such that 1 goes to $q_{1} \ominus|w|$ on $b^{\ell}$. Let

$$
\mathcal{S}^{\prime}=\left\{\left\{0, q_{2} \ominus|w| \ominus \ell\right\} \cup S_{2} \ominus|w| \ominus \ell, \ldots,\left\{0, q_{k} \ominus|w| \ominus \ell\right\} \cup S_{k} \ominus|w| \ominus \ell\right\},
$$

where the operation $\ominus$ is understood to have left-associativity. Then $\mathcal{S}^{\prime}$ is reachable by induction. On $c$, every set $\left\{0, q_{i} \ominus|w| \ominus \ell\right\} \cup S_{i} \ominus|w| \ominus \ell$ goes to the accepting state $\left\{n-1, q_{i} \ominus|w| \ominus \ell\right\} \cup S_{i} \ominus|w| \ominus \ell$ in the NFA $N_{3}$, and therefore also to the initial state $\{0\}$. Then, on $d$, every state $\left\{n-1, q_{i} \ominus|w| \ominus \ell\right\} \cup S_{i} \ominus|w| \ominus \ell$ goes to the rejecting state $\left\{0, q_{i} \ominus|w| \ominus \ell\right\} \cup S_{i} \ominus|w| \ominus \ell$, while $\{0\}$ goes to $\{0,1\}$. Hence, in the DFA $D_{3}$ we have

$$
\begin{aligned}
\mathcal{S}^{\prime} & \stackrel{c}{\rightarrow}\left\{\{0\},\left\{n-1, q_{2} \ominus|w| \ominus \ell\right\} \cup S_{2} \ominus|w| \ominus \ell, \ldots,\left\{n-1, q_{k} \ominus|w| \ominus \ell\right\} \cup S_{k} \ominus|w| \ominus \ell\right\} \\
& \stackrel{d}{\rightarrow}\left\{\{0,1\},\left\{0, q_{2} \ominus|w| \ominus \ell\right\} \cup S_{2} \ominus|w| \ominus \ell, \ldots,\left\{0, q_{k} \ominus|w| \ominus \ell\right\} \cup S_{k} \ominus|w| \ominus \ell\right\} \\
& \stackrel{b^{\ell}}{\rightarrow}\left\{\left\{0, q_{1} \ominus|w|\right\},\left\{0, q_{2} \ominus|w|\right\} \cup S_{2} \ominus|w|, \ldots,\left\{0, q_{k} \ominus|w|\right\} \cup S_{k} \ominus|w|\right\} \stackrel{w}{\rightarrow} \mathcal{S} .
\end{aligned}
$$

It follows that $\mathcal{S}$ is reachable in the DFA $D_{3}$. This concludes the proof.

The next lemma shows that some rejecting states of the DFA $D_{3}$, in which no set is a subset of some other set, may be pairwise distinguishable. To prove the result it uses four symbols, one of which is the symbol $b$ from the proof of the previuos lemma. 


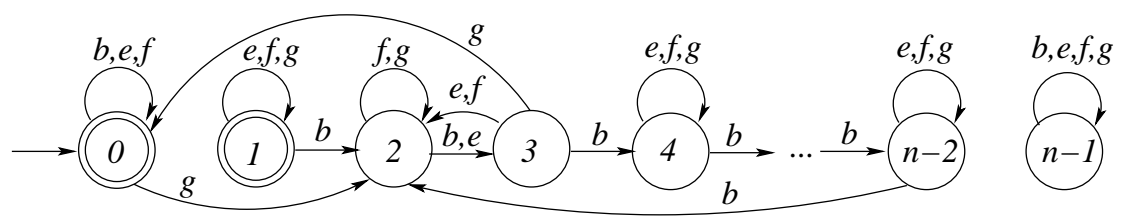

Fig. 6. DFA $D$ over $\{b, e, f, g\}$ with many distinguishable states in DFA $D_{3}$.

Lemma 4. Let $n \geq 5$. There exists an $n$-state DFA $D=\left(Q_{n}, \Sigma, \delta, 0,\{0,1\}\right)$ over a four-letter alphabet $\Sigma$ such that all the states of the DFA $D_{3}$ for the language $L(D)^{+c+}$ of the form

$$
\left\{\{0\} \cup T_{1},\{0\} \cup T_{2}, \ldots,\{0\} \cup T_{k}\right\},
$$

in which no set is a subset of some other set and each $T_{i} \subseteq\{2,3, \ldots, n-2\}$, are pairwise distinguishable.

Proof. To prove the lemma, we reuse the symbol $b$ from the proof of Lemma 3 , and define three new symbols $e, f, g$ as shown in Fig. 6]

Notice that on states $2,3, \ldots, n-2$, the symbol $b$ performs a big permutation, while $e$ performs a trasposition, and $f$ a contraction. It follows that every transformation of states $2,3, \ldots, n-2$ can be performed by strings over $\{b, e, f\}$. In particular, for each subset $T$ of $\{2,3, \ldots, n-2\}$, there is a string $w_{T}$ over $\{b, e, f\}$ such that in $D$, each state in $T$ goes to state 2 on $w_{T}$, while each state in $\{2,3, \ldots, n-2\} \backslash T$ goes to state 3 on $w_{T}$. Moreover, state 0 remains in itself while reading the string $w_{T}$. Next, the symbol $g$ sends state 0 to state 2 , state 3 to state 0 , and state 2 to itself.

It follows that in the NFA $N_{3}$, the state $\{0\} \cup T$, as well as each state $\{0\} \cup T^{\prime}$ with $T^{\prime} \subseteq T$, goes to the accepting state $\{2\}$ on $w_{T} \cdot g$. However, every other state $\{0\} \cup T^{\prime \prime}$ with $T^{\prime \prime} \subseteq\{2,3, \ldots, n-2\}$ is in a state containig 0 , thus in a rejecting state of $N_{3}$, while reading $w_{T} \cdot g$, and it is in the rejecting state $\{0,3\}$ after reading $w_{T}$. Then $\{0,3\}$ goes to the rejecting state $\{0,2\}$ on reading $g$.

Hence the string $w_{T} \cdot g$ is accepted by the NFA $N_{3}$ from each state $\{0\} \cup T^{\prime}$ with $T^{\prime} \subseteq T$, but rejected from any other state $\{0\} \cup T^{\prime \prime}$ with $T^{\prime \prime} \subseteq\{2,3, \ldots, n-2\}$.

Now consider two different states of the DFA $D_{3}$

$$
\begin{aligned}
& \mathcal{T}=\left\{\{0\} \cup T_{1}, \ldots,\{0\} \cup T_{k}\right\}, \\
& \mathcal{R}=\left\{\{0\} \cup R_{1}, \ldots,\{0\} \cup R_{\ell}\right\},
\end{aligned}
$$

in which no set is a subset of some other set and where each $T_{i}$ and each $R_{j}$ is a subset of $\{2,3, \ldots, n-2\}$. Then, without loss of generality, there is a set $\{0\} \cup T_{i}$ in $\mathcal{T}$ that is not in $\mathcal{R}$. If no set $\{0\} \cup T^{\prime}$ with $T^{\prime} \subseteq T_{i}$ is in $\mathcal{R}$, then the string $w_{T_{i}} \cdot g$ is accepted from $\mathcal{T}$ but not from $\mathcal{R}$. If there is a subset $T^{\prime}$ of $T_{i}$ such that $\{0\} \cup T^{\prime}$ is in $\mathcal{R}$, then for each suset $T^{\prime \prime}$ of $T^{\prime}$ the set $\{0\} \cup T^{\prime \prime}$ cannot be in $\mathcal{T}$, and then the string $w_{T^{\prime}} \cdot g$ is accepted from $\mathcal{R}$ but not from $\mathcal{T}$. 
Corollary 2 (Star-Complement-Star: Lower Bound). There exists a language $L$ accepted by an $n$-state DFA over a seven-letter input alphabet, such that any DFA for the language $L^{* c *}$ has $2^{\Omega(n \log n)}$ states.

Proof. Let $\Sigma=\{a, b, c, d, e, f, g\}$ and $L$ be the language accepted by $n$-state DFA $D=(\{0,1, \ldots, n-1\}, \Sigma, \delta, 0,\{0,1\})$, where transitions on symbols $a, b, c, d$ are defined as in the proof of Lemma 3 and on symbols $d, e, f$ as in the proof of Lemma 4.

Let $m=\lceil n / 2\rceil$. By Lemma 3 , the following states are reachable in the DFA $D_{3}$ for $L^{+c+}$ :

$$
\left\{\{0,2\} \cup S_{1},\{0,3\} \cup S_{2}, \ldots,\{0, m-2\} \cup S_{m-1}\right\},
$$

where $S_{1} \subseteq S_{2} \subseteq \cdots \subseteq S_{m-1} \subseteq\{m-1, m, \ldots, n-2\}$. The number of such subsets $S_{i}$ is given by $m^{n-m}$, and we have

$$
m^{n-m} \geq\left(\frac{n}{2}\right)^{\frac{n}{2}-1}=2^{\Omega(n \log n)} .
$$

By Lemma 4, all these states are pairwise distinguishable, and the lower bound follows.

Hence we have an asymptotically tight bound on the state complexity of star-complement-star operation that is significantly smaller than $2^{2^{n}}$.

Theorem 1. The state complexity of star-complement-star is $2^{\Theta(n \log n)}$.

\section{Applications}

We conclude with an application.

Corollary 3. Let $L$ be a regular language, accepted by a DFA with $n$ states. Then any language that can be expressed in terms of $L$ and the operations of positive closure, Kleene closure, and complement has state complexity bounded by $2^{\Theta(n \log n)}$.

Proof. As shown in [1, every such language can be expressed, up to inclusion of $\varepsilon$, as one of the following 5 languages and their complements:

$$
L, L^{+}, L^{c+}, L^{+c+}, L^{c+c+} .
$$

If the state complexity of $L$ is $n$, then clearly the state complexity of $L^{c}$ is also $n$. Furthermore, we know that the state complexity of $L^{+}$is bounded by $2^{n}$ (a more exact bound can be found in [7]); this also handles $L^{c+}$. The remaining languages can be handled with Theorem 1 . 


\section{References}

1. Brzozowski, J., Grant, E., and Shallit, J.: Closures in formal languages and Kuratowski's theorem, Int. J. Found. Comput. Sci. 22, 301-321 (2011)

2. Kleitman, D. and Markowsky, G.: On Dedekind's problem: the number of isotone Boolean functions. II, Trans. Amer. Math. Soc. 213, 373-390 (1975)

3. Rabin, M., Scott, D.: Finite automata and their decision problems. IBM Res. Develop. 3, 114-129 (1959)

4. Sipser, M.: Introduction to the theory of computation. PWS Publishing Company, Boston (1997)

5. Sloane, N. J. A.: Online Encyclopedia of Integer Sequences, http://oeis.org

6. Yu, S.: Chapter 2: Regular languages. In: Rozenberg, G., Salomaa, A. (eds.) Handbook of Formal Languages - Vol. I, pp. 41-110. Springer, Heidelberg (1997)

7. Yu, S., Zhuang, Q., Salomaa, K.: The state complexity of some basic operations on regular languages. Theoret. Comput. Sci. 125, 315-328 (1994) 